\section{Entropy as a method to investigate complex biological systems. An alternative view on the biological transition from healthy aging to frailty}

\author{
Roberto Siciliano \\ Medical Physics Unit, Cosenza Hospital; \\ Adjunct professor in Applied Physics, \\ Magna Graecia University, Catanzaro, \\ Italy
}

\begin{abstract}
Everyone is subject to a process of progressive deterioration of control mechanisms, which supervise the complex network of human physiological functions, reducing the individual ability to adapt to emerging situations of stress or change. In the light of results obtained during the last years, it appears that some of the tools of nonlinear dynamics, first developed for the physical sciences are well suited for studies of biological systems.

We believe that, considering the level of order or complexity of the anatomical apparatus by measuring a physical quantity, which is the entropy, we can evaluate the health status or vice versa fragility of a biological system.
\end{abstract}

In particular, a reduction in the entropy value, indicates modification of the structural order with a progressive reduction of functional reserve of the individual, which is associated with a failure to adapt to stress conditions, difficult to be analyzed and documented with a unique traditional biochemical or biomolecular vision.

Therefore, in this paper, we present a method that, conceptually combines complexity, disease and aging, alloys Poisson statistics, predictive of the personal level of health, to the entropy value indicating the status of bio-dynamic and functional body, seen as a complex and open thermodynamic system.

\section{Introduction}

The continuous adaptations to the needs of the environment that welcomes us, force everyone to face them and manage through complex interactions of physiological control mechanisms that take place and interact at the subcellular, cellular, histological and organ level: the right functioning of this interaction network is the basis of a state of good health. Today aging is described as a process by which it produces a progressive deterioration of the control mechanisms that oversee the complex network of physiological functions; in other words, it can be described as a reduced ability of the individual to adapt to emerging situations of stress or changes. Therefore, the aging of an individual is usually a consequent accumulation of health problems expressible as laboratory values over the threshold of normality, disability, sensory or cognitive symptoms, which generally can be called deficit. If the aging population builds up, as a general rule, a series of deficits, the individual increases its own number of health problems, with a personal frequency.

In recent years, the concept of frailty has found its place in the international community to reflect better the risk of compromised health status of an individual.

Although its operational definition remains controversial in some ways, ${ }^{1,2}$ it is sufficiently accepted that fragility is not intended in itself as a disease state, but an increase in the probability of the realization of a disease, which is highly associated with variation of the complex physiological system.

Frailty is not only a disease sensu stricto, but also an intermediate state between a functional state and a non-functional one, and between a state of health and a disease. ${ }^{2}$

Other authors, ${ }^{3-6}$ have attempted to link the individual fragility to a number of deficits accumulated in the course of its existence.

In past years many scientists have believed that living systems, as also other fields of study, could be understood by application of a reductionist approach. There are strong grounds for this conviction: reductionism has had tremendous success in recent decades in many other scientific disciplines, in particular, the idea was to dissect the various parts of the physical or biological systems considered entirely too complex. But, despite the identification and the characterization of subsets of the system under study, the full understanding of the biological system has not reached, and it is now clear that we must have an integrated point of view, a model to explain the whole phenomenon.

We believe that it is possible a more precise approximation to the real state of health or aging of an individual, assessing the level of order or complexity of the anatomical apparatus through the evaluation of Entropy. Therefore, in this paper, we present our method that links a Poisson statistics, which predicts the level of personal
Correspondence: Roberto Siciliano, Medical Physics Unit, Cosenza Hospital; Adjunct professor in Applied Physics, Magna Graecia University, Catanzaro - Italy.

E-mail: robsic65@gmail.com

Key words: Aging; fractals; entropy; nonlinear dynamics.

Acknowledgements: we thank Prof. A. Mastroberardino for collaboration on reading manuscript.

Received for publication: 18 April 2017. Accepted for publication: 3 July 2017.

This work is licensed under a Creative Commons Attribution-NonCommercial 4.0 International License (CC BY-NC 4.0).

(C) Copyright R. Siciliano, 2017

Licensee PAGEPress, Italy

Geriatric Care 2017; 3:6755

doi:10.4081/gc.2017.6755

vulnerability, to the value of entropy indicating the status of biodynamic and functional body.

\section{Complexity: health, aging and disease}

The result of the aging process, as discussed earlier, is a continuous deterioration of control systems of the human body, which are present at different levels: molecular, cellular, organ and systemic. These control mechanisms govern the complex network of physiological functions.

These processes are dynamic and continuous, so that the body is ready to respond immediately to any type of stress, both external and internal to the body; ${ }^{8,9}$ this understanding has allowed the evolution of the concept of homeostasis to the new concept of oleodynamic, ${ }^{10}$ introduced by Yates, ${ }^{11}$ who inaugurated the idea that the sophisticated control required by the body is possible through the dynamic and continuous interaction of several mechanisms of regulation and control.

Quantifying the complexity of physiological signals of an individual suffering from a disease or in health has been the focus of considerable attention in recent years.Recently, new concepts have been developed to describe the dynamics of physiological systems in order to distinguish different levels of health and even to predict possible future health states. ${ }^{12-14}$

The majority of these methods is derived from methods used in physics in the field of nonlinear dynamics (chaos theory) and statistical physics and use the concept of fractals ${ }^{15-16}$ to try to understand, quantify 
and model the aging of human body, seen as a variation of the complexity of physiological dynamics.

In recent years, it has been observed that different physiological processes have fractal properties of self-similarity (both temporal and spatial) such as heart rate, the anatomy of the respiratory tract, the vascular system and, above all, the intricate network of the neurological system; ${ }^{17}$ therefore, it is becoming clear that it is necessary to consider them as complex systems and the link between them, and their modifications in time, cannot be fully understood without using new techniques.

Indeed, complex systems can give rise to collective behaviors, which are not simply the sum of their individual components ${ }^{18}$ but involve huge conglomerations of related units constantly interacting with their environment. The way in which this happens is still a mystery. Understanding the emergence of ordered behavior of spatio-temporal patterns and adaptive functions appears to require additional, and more global, concepts and tools.

A useful method for evaluating the complexity of a system is to calculate the entropy. This method is conceptually linked to the classical physics of a thermodynamic system and indicates the degree of overall order of the molecules that compose it.

\section{Materials and Methods}

\section{Entropy}

Contrary to its common usage, the mathematical sense of the word chaos does not mean disorder or confusion. It indicates a specific system with dynamical behavior. Several methods and measures are available to characterize chaotic systems. Despite the sensitivity of these systems to initial conditions and the rapid divergence in their evolution, some of these measures are invariant, in the sense that their results do not depend from the starting point of the system. An important invariant physical quantity is entropy.

Entropy is a thermodynamic quantity describing the amount of order in a system, and it supplies an important approach for the analysis of a system that evolves in the time, which can be regarded as a source of information. From a microscopic point of view, the second law of thermodynamics tells us that a system tends to evolve towards a condition, which has the largest number of accessible states compatible with the macroscopic conditions. Maximum entropy corresponds to the maximum number of possible microscopic positions (high fractality).
In a state space, which is the first step for characterizing the behavior of systems and their variations in time, current research in this field goes by the name of dynamical systems theory. The entropy of a system $\mathrm{S}(\mathrm{x})$ of a random variable $\mathrm{x}$, can then be written: ${ }^{19}$

$$
S(x)=-\sum_{x_{i} \in \Theta} p\left(x_{i}\right) \ln p\left(x_{i}\right)
$$

where $\mathrm{x}$ is a random variable with values in $\mathrm{Q}$, and represents the set of possible events (in our case: the set of possible health states) with probability function $\mathrm{p}\left(\mathrm{x}_{\mathrm{i}}\right)$ to denote the probability of the $\mathrm{i}$-th event. Using a Markov approach, $\mathrm{p}\left(\mathrm{x}_{\mathrm{i}}\right)$ shall be understood as the conditional probability of its recent past, that is: $\mathrm{p}\left(\mathrm{x}_{\mathrm{i}} / \mathrm{x}_{\mathrm{i}-1}\right)$.

Clearly, the generic system state $\mathrm{x}_{\mathrm{i}}$ is influenced by states that are part of the past, indicated by the variables $\mathrm{x}_{1}, \mathrm{x}_{2}, \ldots, \mathrm{x}_{\mathrm{i}-1}$.

The value of nonlinear entropy is now seen as the measure of information that is missing or needed to positively identify a particular state of the system under investigation, described by a set of stochastic processes, each of which consists of $\mathrm{n}$ random variables $\mathrm{x}_{\mathrm{i} \cdot}{ }^{16,19,20}$

\section{Results}

\section{Levels of entropic health}

In recent years, many researchers and clinicians investigating human aging seen as a loss of complexity and fractality of anatomical structures and physiological processes, have used the concept of entropy to quantify changes in fractality or irregularities in the dynamics of physiological systems. ${ }^{19-21}$

These studies showed clearly that a state of health is described by a state of normal irregularity (chaotic state), while the disease or old age would be characterized by an increase in regularity, for example, much more regular EEG during an epileptic seizure, heart rate and ECG in more regular people who are sick or elderly, some forms of leukemia are preceded by a regularization of the production of white blood cells.

To quantify, therefore, the nonlinear value of the entropy of a chaotic biological system, is equivalent to measure the complexity of the dynamical system examined.

Recent studies have shown that the distribution of the state of health of a community of people can be investigated using the Poisson statistical law. In fact, this statistical method, was used to assess the probability of an individual to transition into a state of frailty different from that of departure. ${ }^{22-}$ ${ }^{24}$ In this case, health is defined as a macro- scopic level of welfare, which is detected by biochemical tests and clinical visits.If we consider the Poisson distribution as a valid conceptual model to describe the evolution of the state of health of a particular organ or whole body, this paper aims to describe the order level of a biological system using the state function entropy.

Therefore, we may consider a biological dynamical system and for simplicity only one biodynamic signal (e.g., EEG), with numeric value of the initial state defined from $S_{n}$, the probability of transitions between two states is:

$P_{n x}=\frac{S_{n}^{x}}{x !} K_{n}$

where $\mathrm{x}$ represents the numeric value of a generic state of health final. $\mathrm{K}_{\mathrm{n}}$ is a positive parameter that depend from the actual health state $\mathrm{n}$, so for every $\mathrm{x}$ we have the conditions that:

$\sum_{x} \frac{S_{n}^{x}}{x !} K_{n}=1$

Then:

$$
K_{n}=\frac{1}{\sum_{x}\left(S_{n}^{x} / x !\right)}
$$

whereas $\mathrm{x}$ changes from 0 to infinity, the formula (4) can be simplified as follows:

$$
K_{n}=e^{-S_{n}}
$$

therefore:

$$
P_{n x}=\frac{S_{n}^{x}}{x !} e^{-S_{n}}
$$

Equation (6) represents the probability that a biological system as a whole, with an initial level of order (n), or similarly of complexity, indicated by the value of entropy $S_{n}$, undergoes a transition to a different state of the final complex (x) indicated by the value of entropy $S_{x}$.

Through the equation (6), we can, in a more precise way, it is possible to define the future probabilities to transit to a different level of physical performance specified by a defined value of entropy. The state of health is now defined exclusively from the individual assessment of biodynamic data processing.

The analysis of biodynamic signals that provide information of early levels of the disease process (functional level), is a level of information earlier than that considered by conventional medicine (biochemical and anatomical data). 


\section{Discussion}

\section{Entropy landscapes}

In the theory of dynamical systems, the concept of stability describes the behavior of a generic system that varies around its position of equilibrium.

It is intuitive to represent a point of stable equilibrium as a position that does not feel small perturbations, i.e., if the system is slightly away from the point of equilibrium, it will continue to remain permanently in the vicinity of that point. Similarly, a system in equilibrium is called unstable when subject to a small perturbation that removes it even slightly from its equilibrium position, tends to distance itself even more.

A simple example to describe a system in stable or unstable equilibrium, is to imagine the system as a ball. If it is placed on the top of a hill, it is in a state of unstable equilibrium, whereas if it is positioned in the bottom of a valley, removed from its position, it can roll or oscillate, but does not go away from its initial position of stable equilibrium (Figure 1).

The metaphor of the landscape is present in the literature and is used to describe the evolution of a physical system in relation to the change of possessed potential energy. In all spontaneous phenomena, according to the second law of thermodynamics, we can see that the system goes towards configurations of minimal potential energy and thus greater stability (Figure 2).

A biological organism is always a physical system and, as such, must follow the general laws of physics. Such an approach, has been used in the context of cellular differentiation, in the picturesque vision of an epigenetic landscape. ${ }^{25,26}$

Statistical thermodynamics predicts that in a biological system (which must neces- sarily be seen as an open system), the state of equilibrium will be reached in accordance with the principle of maximum entropy and the principle of minimum energy; therefore, the final stable configuration is that one that will balance the two opposing needs to minimize energy and maximize entropy.

If we consider the dynamics of the physiological system of a young and healthy individual, so to consider it as a reference condition to indicate a state of stable equilibrium, we may associate with this condition the corresponding values of energy (minimum energy condition or energy landscape) and entropy (maximum entropy value) of the biological system. That is, the system resides in a particular equilibrium state of minimum as possible energy and at the same time expresses the maximum possible entropy of the complex physiological dynamics, in analogy with every phenomenon that occurs in the physical world, which is trying to reach its equilibrium state maximizing results and minimizing energy waste. Among all the equilibrium states allowed, the one just described, which is selected by nature, is the best possible state of equilibrium at a given time attributable to a particular physical performance.

We believe that every individual passes through the time in a succession of stable conditions, macroscopically evaluated through the value of the entropy and with the probability of occurrence as calculated according to equation (6).

The physiological system of an individual with a poor health, diseased, or simply aged, which manifests itself through the regularization of physiological processes resulting in reduction of entropy, as a result of the second law of thermodynamics, must necessarily increase energy, moving toward a state of instability.
In the metaphor of the landscape, this condition can be located on the top of the hill (Figure 1) but at the same time, the set of physiological control mechanisms generates forces that promote the system to a new state of stable equilibrium.

In other words, it is as if the complex dynamic system foresaw the existence of multiple physiological levels of stability (multistability) ${ }^{27,28}$ perturbed by biological attractors that are always trying to direct the evolution of the chaotic and unpredictable biological system to equilibrium conditions thermodynamically stable (Figure 3).

If the biological attractors succeed in their goal of leading the system into a state of stable equilibrium, at high entropy value, then the individual experiences health conditions, or conditions that may bring to a disease state or a reduced ability to react to new stresses.

The concept that, at any given moment, the general condition of the organism is the result of the synergic action of the control mechanisms that regulate the complex network of physiological functions, is adherent to the notion of multistability of a system as a result of the underlying dynamic system. This concept was first suggested, in relation to cell differentiation, by the Nobel prize for physics M. Delbrück ${ }^{27}$ and subsequently taken up and extended by other researchers. ${ }^{28-30}$

This approach suggests that a biological organism as a whole, due to internal or external stress (i.e., induced by the dynamic physiological or environmental factors), transits over time in different health states conditioned by biological attractors that seek to influence the possible transitions favoring those that converge towards a final state which has both the minimum potential energy and the maximum possible entropy.

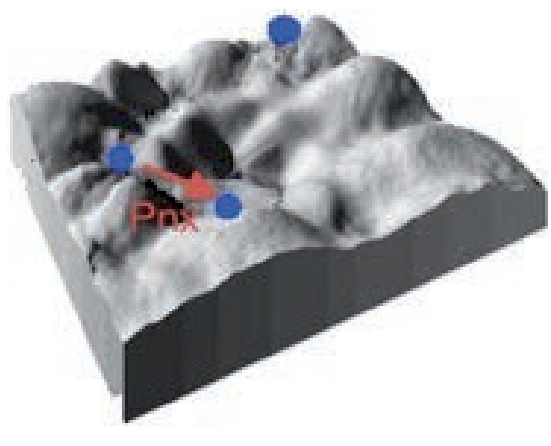

Figure 1. Schematic representation of an energy landscape with points equilibrium (valley) and instability points (hills) separated from energy barriers.

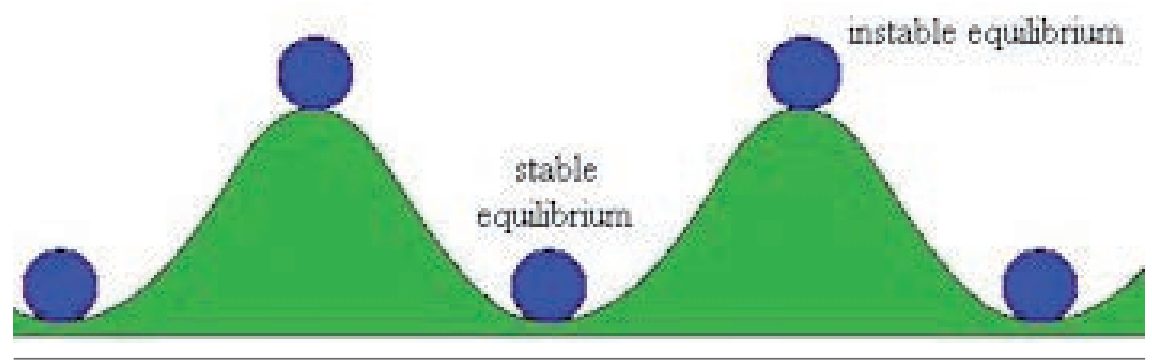

Figure 2. Energy landscape with plains separated from energy barriers. The balls indicate particular health levels. 


\section{Conclusions}

Recently, several working groups are projected in research to quantify and understand the physiological signals of an individual, either during a state of health or disease. They are currently proposing new models to assess the complex dynamics of biological control systems, which aging and pathological events tend to degrade.

This degradation is manifested by a different physiological signals output (loss of complexity), indicating a reduced ability to adapt the organism, namely the establishment of a potentially pathological condition.

This general state of physiological decline may be attributed to altered activity or function of an organ or to an altered physiological dynamics. disease, aging and complexity, suggests new methods for understanding the general mechanisms such as the measure of entropy. This hypothesis, in order to assess the real performance level of the organism and make possible to take appropriate actions (physical, pharmacological) aims at combating, as far as possible, the physiological

In this paper is presented a general model for understanding a potential future state of health as a function of the output biodynamic signals described as changes in the values of entropy. The model shows that, knowing the value of the entropy, exists the possibility of improving the understanding of how to evolve in the short-term health status of a biological organism. Such knowledge is early compared to those who can supply the traditional methods of investigation.
The conceptual hypothesis that links process in place.
We believe it is the right way to go to improve the general understanding of the physiological system of the human organism, by adopting new models and experimental paradigms, such as those of fractality and entropy, which tend to focus on knowledge from an organ medicine to a systemic medicine.

\section{References}

1. Bortz WM II. A conceptual framework of frailty: a review. J Gerontol Med Sci 2002;57A:M283-8.

2. Fried LP, Tangen CM, Walston J, et al. Frailty in older adults. J Gerontol Med Sci 2001;56A:M146-56.

3. Goggins WB, Woo J, Sham A, Ho SC. Frailty index as a measure of personal biological age in a Chinese population. J Gerontol A Biol Sci Med Sci 2005;60A:1046-51.

4. Rockwood K, Mitnitski A. Frailty in relation to the accumulation of deficits. J Gerontol Med Sci 2007;62A:722-7.

5. Woo J, Goggins W, Sham A, Ho SC. Public health significance of the frailty index. Disabil Rehabil 2006;28:515-21.

6. Kulminski A, Yashin A, Arbeev K, et al. Cumulative index of health disorders as an indicator of aging-associatied processes in the elderly: results from analyses of the National Long Term Care Survey. Mech Ageing Dev 2007; 128:250-8.

7. Palazzo S, Siciliano R, Turano S. Neoplasie dell'anziano - cap. 4. Lecce: Ed. Milella; 2010.

8. Kirkwood TB. Understanding the odd science of aging. Cell 2005;120:437-47.

9. Wilson DM III, Bohr VA, McKinnon PJ. DNA damage, DNA repair, ageing and age-related disease. Mech Ageing Dev 2008;129: 349-52.

10. Lipsitz LA. Dynamics of stability: the physiologic basis of functional health and frailty. J Gerontol Biol Sci 2002; 57A:B115-25.

11. Yates FE. Self-organizing systems. In: Boyd CAR, Noble R, eds. The logic of life - the challenge of integrative physiology. New York: Oxford University Press; 1993. pp 189-218.

12. Hortelano M, Reilly RB, Cervigòn R. Multiscale entropy analysis of heart rate regularity changes in older people with orthostatic intolerance. XIV Mediterra-nean Conference. Med Biol Engine Comput 2016 [Epub ahead of print].

13. Fischer R, Akay M, Castiglioni P, Di Rienzo M. Multi-and monofractal indices of short-term heart rate variability. Med Biol Engine Comput 2003;41: 543-9.

14. Cuesta D, Varela M, Miro P, et al. Predicting survival in critical patients by use of body temperature regularity measurement based on approximate entropy. Med Biol Engine Comput 2007;45:671-8.

15. Mandelbrot BB. The fractal geometry of nature. New York: Freeman; 1983.

16. Peng CK, Havlin S, Stanley HE, Goldberger AL. Quantification of scaling exponents and crossover phenomena in nonstationary heartbeat time series. Chaos 1995;5:82-7.

17. Li P, Liu CY, Sun X, et al. Assessing the complexity of short-term heartbeat interval series by distance distribution entropy. Med Biol Engine Comput 2015;53:77-87.

18. Anderson PW. More is different. Science 1972;177:393-6.

19. Costa M. Multiscale entropy analysis of biological signals. Phys Rev 2005;71: 021906.

20. Costa M, Goldberger AL, Peng C-K. Multiscale entropy analysis of complex physiological time series. Phys Rev Lett 2002;89:068102.

21. Lipsitz LA. Physiological complexity, aging, and the path to frailty. Sci Aging Knowl Environ 2004;16:16.

22. Mitnitski AB, Song X, Rockwood KJ. The estimation of relative fitness and frailty in community-dwelling older adults using self-report data. J Gerontol Med Sci 2004;59A:627-32.

Figure 3. Generic representation of an energy landscape and its associated hill (energy barriers). Local minima (equilibrium points) are labeled with $e_{1-5}$, saddle points (instability points) are marked with $i_{1-4}$
23. Mitnitski A, Bao L, Rockwood K. Going from bad to worse: a stochastic model of transitions in deficit accumu- 
lation, in relation to mortality. Mechan Ageing Develop 2006;127:490-3.

24. Goggins WB, Woo J, Sham A, Ho SC. Frailty index as a measure of personal biological age in a Chinese population. J Gerontol A Biol Sci Med Sci 2005;60:1046-51.

25. Waddington $\mathrm{CH}$. The strategy of the genes. London: Allen and Unwin; 1957.
26. Goldberg AD, Allis CD, Bernstein E. Epigenetics: a landscape takes shape. Cell 2007;128:635-8.

27. Delbrück M. Unités biologiques douées de continuité génétique. Paris: Editions du Centre National de la Recherche Scientifique; 1949. Discussion pp 33-35.

28. Kauffman, S. The origins of order: Selforganization and selection in evolution.
New York: Oxford University Press; 1993.

29. Thomas R. Laws for the dynamics of regulatory networks. Int $\mathrm{J}$ Dev Biol 1998;42:479-85.

30. MacArthur BD, Please CP, Oreffo ROC. Stochasticity and the molecular mechanisms of induced pluripotency. PLoS One 2008;3:e3086. 